\title{
AN INTEGRAL INEQUALITY
}

\author{
J. ERNEST WILKINS, JR.
}

(Communicated by R. Daniel Mauldin)

\begin{abstract}
We furnish conditions on the functions $p(t), f(t)$, and $g(t)$ that are sufficient for the validity of the inequality, $\alpha^{2} \delta \geq \gamma^{2} \beta$, in which $\alpha, \beta$, $\gamma$, and $\delta$ respectively, are the integrals over a measurable set $E$ of $p(t) g(t)$, $p(t) g^{2}(t), p(t) f(t)$, and $p(t) f^{2}(t)$.
\end{abstract}

\section{INTRODUCTION}

In this paper we consider conditions that assure the validity of the inequality,

$$
\alpha^{2} \delta-\gamma^{2} \beta \geq 0,
$$

in which

$$
\begin{aligned}
\alpha & =\int_{E} p(t) g(t) d t, & & \beta=\int_{E} p(t) g^{2}(t) d t, \\
\gamma & =\int_{E} p(t) f(t) d t, & \delta & =\int_{E} p(t) f^{2}(t) d t .
\end{aligned}
$$

In spite of its simplicity and its resemblance to the Tchebycheff inequality [4, Theorem 10, p. 40], this inequality does not appear to have been discussed in the standard treatises on inequalities [2-4]. Our interest in (1) was triggered by the special case [6] in which $E=(0,1), p(t)=t, g(t)=J_{0}(v t), f(t)=t^{2} J_{0}^{\prime \prime}(v t)$, $v$ is a fixed constant, and $J_{0}$ is the Bessel function of the first kind and order zero.

We always assume that $E$ is a measurable set with positive measure, that the real-valued function $p(t)$ is integrable on $E$, and that the real-valued functions $g(t)$ and $f(t)$ are bounded and measurable on $E$. Consequently, the four integrals in (2) exist.

In $\S \S 2,4$ we prove the following two theorems, each of which states conditions sufficient for the validity of (1).

Received by the editors September 6, 1989.

1980 Mathematics Subject Classification (1985 Revision). Primary 26D15.

Key words and phrases. Inequalities, integral inequalities. 
Theorem 1. The inequality (1) is true if

$$
\begin{aligned}
p(s) p(t) p(u)[ & g^{2}(s)\{f(t)-f(u)\}^{2}+g^{2}(t)\{f(u)-f(s)\}^{2} \\
& +g^{2}(u)\{f(s)-f(t)\}^{2}-f^{2}(s)\{g(t)-g(u)\}^{2} \\
& \left.-f^{2}(t)\{g(u)-g(s)\}^{2}-f^{2}(u)\{g(s)-g(t)\}^{2}\right] \geq 0
\end{aligned}
$$

a.e. on $E^{3}=E \times E \times E$. The inequality (1) is strict if (3) holds a.e. on $E^{3}$ and is strict on a measurable subset of $E^{3}$ whose measure is positive. Equality a.e. on $E^{3}$ in (3) implies that

$$
\alpha p(t) f(t)=\gamma p(t) g(t) \quad \text { a.e. on } E ;
$$

this condition implies equality in (1), and in (3) if $(\alpha, \gamma) \neq(0,0)$.

Theorem 2. The inequality (1) is true if

$$
p(t) p(u)\{g(t) f(u)-g(u) f(t)\}\{h(u)-h(t)\} \geq 0
$$

a.e. on $E^{2}=E \times E$, in which $h(t)=\alpha f(t)+\gamma g(t)$. The inequality (1) is strict if

(5) holds a.e. on $E^{2}$ and is strict on a measurable subset of $E^{2}$ whose measure is positive. Equality a.e. on $E^{2}$ in (5) occurs if and only if either (4) holds or

$$
h(t)=\rho \quad \text { a.e. on } E-E_{1} \text {, }
$$

in which $E_{1}$ is the subset of $E$ on which $p(t) f(t)=0, p(t) g(t)=0$, and $\rho$ is a constant.

Because it may be cumbersome to test inequality (3), in $\S 3$ we give a series of corollaries to Theorem 1 with progressively stronger hypotheses that are progressively easier to test. A similarly motivated corollary to Theorem 2 is given at the end of $\S 4$. We note that neither Theorem contains an explicit hypothesis on the sign of $p(t)$. Finally, in $\S 5$ we analyze the special case mentioned in the first paragraph. For this case we infer from Corollary 3 to Theorem 1 and from Corollary 4 to Theorem 2 that (1) holds strictly when $0<v \leq v_{1}$ and when $0<v \leq v_{2}$, respectively, in which $v_{1} \sim 1.0944$ and $v_{2} \sim 1.1668$. The first of these inferences is valid even if $p(t)$ is replaced by an arbitrary integrable and a.e. positive function.

\section{Proof OF TheOREM 1}

Fubini's Theorem and some simple manipulations show that

$$
\begin{aligned}
6\left(\alpha^{2} \delta-\gamma^{2} \beta\right) & =6 \iiint_{E^{3}} p(s) p(t) p(u) g(s) f(u)\{g(t) f(u)-g(s) f(t)\} d s d t d u \\
& =\iiint_{E^{3}} F(s, t, u) d s d t d u
\end{aligned}
$$

in which

$$
\begin{aligned}
F(s, t, u)=2 p(s) p(t) p(u)\{ & g(s) g(t) f^{2}(u)+g(t) g(u) f^{2}(s)+g(u) g(s) f^{2}(t) \\
& \left.-f(s) f(t) g^{2}(u)-f(t) f(u) g^{2}(s)-f(u) f(s) g^{2}(t)\right\} .
\end{aligned}
$$


Because $F$ is equal to the left side of (3), the assertions of Theorem 1 are now obvious, except for the assertions in the last sentence.

It is clear that (4) is a consequence of (3) when $(\alpha, \gamma)=(0,0)$. Assume now that $(\alpha, \gamma) \neq(0,0)$ and that equality occurs a.e. on $E^{3}$ in (3). Then

$$
F(s, t, u)=0 \quad \text { a.e. on } E^{3}, \alpha^{2} \delta-\gamma^{2} \beta=0 .
$$

If we integrate the first equation in (7) with respect to $t$ and $u$ over $E^{2}$, we find that

$$
p(s)\left\{\alpha^{2} f^{2}(s)-\gamma^{2} g^{2}(s)-2 \beta \gamma f(s)+2 \alpha \delta g(s)\right\}=0 \quad \text { a.e. on } E .
$$

If $\gamma=0$, then $\alpha \neq 0, \delta=0$, and (8) implies that $p(s) f^{2}(s)=0$ a.e. on $E$; this is sufficient to guarantee (4). A similar argument disposes of the case when $\alpha=0, \gamma \neq 0$. Henceforth we assume that $\alpha \gamma \neq 0$. Then it follows from the second equation in (7) that we may define $\varepsilon$ as either $\alpha \delta / \gamma$ or $\gamma \beta / \alpha$. We then infer from (8) that

$$
p(s)\{\alpha f(s)-\gamma g(s)\{\{\alpha f(s)+\gamma g(s)-2 \varepsilon\}=0 \quad \text { a.e. on } E .
$$

We define $E_{2}$ as the subset of $E$ on which (9) holds and where $p(s)\{\alpha f(s)-\gamma g(s)\} \neq 0$. If meas $E_{2}=0$, then (4) is surely true. If meas $E_{2}>$ 0 , if follows from (9) that

$$
\begin{gathered}
f(s)=\{2 \varepsilon-\gamma g(s)\} / \alpha \quad \text { on } E_{2}, \\
p(s) f(s)=\gamma p(s) g(s) / \alpha \quad \text { a.e. on } E-E_{2} .
\end{gathered}
$$

For almost all $(s, t, u)$ in $E_{2} \times\left(E-E_{2}\right) \times\left(E-E_{2}\right)$ the first equation in (7) implies, with the help of (10), (11), and a little algebra, that

$$
p(s) p(t) g(t) p(u) g(u)\{\gamma g(s)-\varepsilon\}[\gamma\{g(t)+g(u)\}-2 \varepsilon]=0 .
$$

Because $p(s)\{\gamma g(s)-\varepsilon\}=p(s)\{\gamma g(s)-\alpha f(s)\} / 2 \neq 0$ on $E_{2}$ and meas $E_{2}>0$, it follows that

$$
p(t) g(t) p(u) g(u)[\gamma\{g(t)+g(u)\}-2 \varepsilon]=0 \quad \text { a.e. on }\left(E-E_{2}\right)^{2} \text {. }
$$

Now define $E_{3}$ and $E_{4}$ as the subsets of $E-E_{2}$ on which $p(t) g(t)>0$ and $p(t) g(t)<0$, respectively. First we consider the case in which meas $\left(E_{3}+E_{4}\right)=$ 0 . Then $p(t) g(t)=0$ a.e. on $E-E_{2}$, it follows from (11) that $p(t) f(t)=0$ a.e. on $E-E_{2}$, and we infer from (2) and (10) that

$$
2 \alpha \gamma=\int_{E_{2}} p(t)\{\alpha f(t)+\gamma g(t)\} d t=2 \varepsilon \int_{E_{2}} p(t) d t .
$$

Therefore, $\varepsilon \neq 0$, and it follows from the first equation in (7) after some algebra that

$$
\begin{aligned}
& g^{2}(s)[\gamma\{g(t)+g(u)\}-2 \varepsilon] \\
& \quad+g(s)\left[\gamma\left\{g^{2}(t)-6 g(t) g(u)+g^{2}(u)\right\}+2 \varepsilon\{g(t)+g(u)\}\right] \\
& \quad+\gamma g(t) g(u)\{g(t)+g(u)\}-2 \varepsilon\left\{g^{2}(t)-g(t) g(u)+g^{2}(u)\right\}=0
\end{aligned}
$$


a.e. on $E_{2}^{3}$. Suppose, if possible, that

$$
\gamma\{g(t)+g(u)\}-2 \varepsilon=0 \quad \text { a.e. on } E_{2}^{2} .
$$

An integration with respect to $u$ over $E_{2}$ then shows that

$$
g(t)=(2 \varepsilon / \gamma)-\left(\operatorname{meas} E_{2}\right)^{-1} \int_{E_{2}} g(u) d u \quad \text { a.e. on } E_{2},
$$

so that $g(t)=\varepsilon / \gamma$ a.e. on $E_{2}, f(t)=\varepsilon / \alpha$ a.e. on $E_{2}, \alpha f(t)=\gamma g(t)$ a.e. on $E_{2}$. From this contradiction of the definition of $E_{2}$, we infer that there is a subset $D$ of $E_{2}^{2}$ such that meas $D>0$ and $\gamma\{g(t)+g(u)\}-2 \varepsilon$ is either positive, or is negative, a.e. on $D$. In either case, the result of an integration of (14) over $D$ shows that $g(s)$ is a.e. on $E_{2}$ a solution of a quadratic equation, $A g^{2}+B g+C=0$, in which $A, B$, and $C$ are constants such that $A \neq 0$. If this quadratic equation has unequal roots $g_{1}$ and $g_{2}$, and if the subsets $E_{5}$ and $E_{6}$ of $E_{2}$ on which $g(s)=g_{1}$ and $g(s)=g_{2}$, respectively, both have positive measure, then the first equation in (7) would imply, for almost all $(s, t, u)$ in $E_{5} \times E_{6} \times E_{6}$, that $\left(g_{1}-g_{2}\right)^{2}\left(\gamma g_{2}-\varepsilon\right)=0$, even though neither factor is zero. From this contradiction we infer that $g(s)$ is constant a.e. on $E_{2}$. It follows from (10) that $f(s)$ is also constant a.e. on $E_{2}$, and (4) is a consequence of the identity,

$$
\begin{aligned}
0 & =\int_{E} p(s)\{\alpha f(s)-\gamma g(s)\} d s \\
& =(\alpha f-\gamma g) \int_{E_{2}} p(s) d s+\int_{E-E_{2}} p(s)\{\alpha f(s)-\gamma g(s)\} d s,
\end{aligned}
$$

equation (11), and the implication of (13) that

$$
\int_{E_{2}} p(s) d s \neq 0 \text {. }
$$

Now consider the case in which meas $E_{3}>0$ or meas $E_{4}>0$. If we integrate (12) over $E_{3}$ or $E_{4}$, we find that $g(t)$ is a constant a.e. on $E_{3}+E_{4}$. That constant must be $\varepsilon / \gamma, \varepsilon \neq 0$ by virtue of the definitions of $E_{3}$ and $E_{4}$. It then follows from (11) that $f(t)=\varepsilon / \alpha$ a.e. on $E_{3}+E_{4}$. For almost all $(s, t, u)$ in $\left(E_{3}+E_{4}\right) \times E_{2} \times E_{2}$, the first equation in (7) now shows, with the help of (10) and a little algebra, that

$$
\{\gamma g(t)-\varepsilon)\{\gamma g(u)-\varepsilon\}[\gamma\{g(t)-g(u)\}-2 \varepsilon]=0 .
$$

Because the first two factors do not vanish on $E_{2}$, we see that (15) is true. A repetition of the argument following (15) leads to a contradiction, from which we infer that this case cannot occur.

This completes the proof that (4) is a consequence of equality a.e. on $E^{3}$ in (3). Conversely, it is obvious that equality in (1) is a consequence of (4) when $(\alpha, \gamma)=(0,0)$, and almost as obvious that equality in both (1) and (3) 
is a consequence of $(4)$ when $(\alpha, \gamma) \neq(0,0)$. This completes the proof of Theorem 1.

\section{SOME COROLlaRIES TO THEOREM 1}

We observe that the triple integral over $E^{3}$ of the left side of (3) is the same as

$3 \iiint_{E^{3}} p(s) p(t) p(u)\left[g^{2}(s)\{f(t)-f(u)\}^{2}-f^{2}(s)\{g(t)-g(u)\}^{2}\right] d s d t d u$.

The following corollary now follows at once from the proof of Theorem 1 .

Corollary 1. The inequality (1) is true if

$p(s) p(t) p(u)\left[g^{2}(s)\{f(t)-f(u)\}^{2}-f^{2}(s)\{g(t)-g(u)\}^{2}\right] \geq 0$ a.e. on $E^{3}$

The inequality (1) is strict if (16) holds a.e. on $E^{3}$ and is strict on a measurable subset of $E^{3}$ whose measure is positive. Equality a.e. on $E^{3}$ in (16) implies (4), and is implied by $(4)$ if $(\alpha, \gamma) \neq(0,0)$.

We next state the following corollary.

Corollary 2. Suppose that $p(t)>0$ a.e. on $E$, that the subset of $E^{2}$ on which $g(t)=g(u)$ is a null set, and that

$$
\begin{gathered}
e g l_{(t, u) \in E^{2}}\left[\{f(t)-f(u)\}^{2} /\{g(t)-g(u)\}^{2}\right] \\
\geq \text { ess. l.u.b. } \text { s }_{s \in E}\left[\{f(s) / g(s)\}^{2}\right] .
\end{gathered}
$$

Then the inequality (1) is true, and is strict unless

$$
\alpha f(t)=\gamma g(t) \quad \text { a.e. on } E,
$$

in which case (1) is an equality, and (17) is an equality if $(\alpha, \gamma) \neq(0,0)$.

In order to prove Corollary 2 , we first observe that $g(s) \neq 0$ a.e. on $E$ and that the left side of $(17)$ is finite. Because $p(t)>0$ a.e. on $E$, it follows that (16) is a consequence of (17), and that the truth of (1) is assured by Corollary 1. Moreover, equality in (1) implies the existence of a constant $\mu$ such that

$$
\{f(s) / g(s)\}^{2}=\mu=\{f(t)-f(u)\}^{2} /\{g(t)-g(u)\}^{2} \quad \text { a.e. on } E^{2} .
$$

A little algebra shows that $f(t) f(u)=\mu g(t) g(u)$ a.e. on $E^{2}$. Multiplication by $p(u)$ and integration with respect to $u$ over $E$ yields the result that $\gamma f(t)=$ $\mu \alpha g(t)$ a.e. on $E$. A subsequent multiplication by $p(t)$ an integration with respect to $t$ over $E$ shows that $\gamma^{2}=\mu \alpha^{2}$. The validity of (18) when $\gamma \neq 0$ is now obvious. If $\gamma=0$, then either $\alpha=0$, in which case (18) is surely true, or $\mu=0$, in which case it follows from (19) that $f(s)=0$ a.e. on $E$, so that (18) is surely true. Finally, it is clear when $(\alpha, \gamma)=(0,0)$ that equality in (1) is a consequence of (18); this conclusion is almost as clear when $(\alpha, \gamma) \neq(0,0)$. 
Corollary 3. Suppose that $E$ is the closed interval $(a, b)$, in which $a$ and $b$ are real numbers such that $a<b$, that $p(t)>0$ a.e. on $E$, that $f(t)$ and $g(t)$ are continuously differentiable on $E$, that $g(t) \neq 0$ on $E$, that $g^{\prime}(t) \neq 0$ when $a<t<b$, and that

$$
\min _{x \in E}\left[\left\{f^{\prime}(x) / g^{\prime}(x)\right\}^{2}\right] \geq \max _{s \in E}\left[\{f(s) / g(s)\}^{2}\right] .
$$

Then the inequality (1) is true, and is strict unless

$$
f(t)=(\gamma / \alpha) g(t) \text { on } E \text {. }
$$

Because $g^{\prime}(t) \neq 0$ when $a<t<b$, the function $\phi(t, u)$ defined so that

$$
\begin{aligned}
& \phi(t, u)=\{f(t-f(u)\} /\{g(t)-g(u)\} \quad \text { when } t \neq u, \\
& \phi(t, u)=f^{\prime}(u) / g^{\prime}(u) \quad \text { when } t=u,
\end{aligned}
$$

is lower semi-continuous on $E^{2}$. The function $f(s) / g(s)$ is continuous on $E$. We can now replace ess. g.l.b. and ess. l.u.b. in (17) with min and max. The inequalities (17) and (20) are equivalent because $\phi(t, u)=f^{\prime}(x) / g^{\prime}(x)$ for some $x$ between $t$ and $u$. Corollary 3 is now a consequence of Corollary 2 and the observation that $\alpha \neq 0$.

\section{Proof of Theorem 2 AND a COROLlary}

The following calculation is sufficient to establish all of Theorem 2 except its last sentence.

$$
\begin{aligned}
2\left(\alpha^{2} \delta\right. & \left.-\gamma^{2} \beta\right)=2 \alpha \int_{E} p(t) g(t) d t \int_{E} p(t) f^{2}(t) d t-2 \gamma \int_{E} p(t) f(t) d t \int_{E} p(t) g^{2}(t) d t \\
= & 2 \alpha\left\{\int_{E} p(t) g(t) d t \int_{E} p(u) f^{2}(u) d u-\int_{E} p(u) f(u) d u \int_{E} p(t) f(t) g(t) d t\right\} \\
& +2 \gamma\left\{\int_{E} p(t) g(t) d t \int_{E} p(u) f(u) g(u) d u-\int_{E} p(u) f(u) d u \int_{E} p(t) g^{2}(t) d t\right\} \\
= & 2 \iint_{E^{2}} p(t) p(u) g(t) f(u)\{h(u)-h(t)\} d t d u \\
= & \iint_{E^{2}} p(t) p(u)\{g(t) f(u)-g(u) f(t)\}\{h(u)-h(t)\} d t d u .
\end{aligned}
$$

It is obvious when $(\alpha, \gamma)=(0,0)$ that equality a.e. on $E^{2}$ in $(5)$ is a consequence of either (4) or (6); this conclusion is almost as obvious when $(\alpha, \gamma) \neq(0,0)$. The converse is obvious when $(\alpha, \gamma)=(0,0)$. When $(\alpha, \gamma) \neq$ $(0,0)$ and equality occurs a.e. on $E^{2}$ in (5), an integration of (5) with respect to $u$ over $E$ shows that

$$
p(t) g(t)\{\alpha \delta+\gamma \zeta-h(t)\}-p(t) f(t)\{\alpha \zeta+\gamma \beta-h(t)\}=0
$$

a.e. on $E$, in which

$$
\zeta=\int_{E} p(u) f(u) g(u) d u
$$


When $\alpha=0, \gamma \neq 0$ (or $\gamma=0, \alpha \neq 0$ ), it follows from equality in (1) that $\beta=0$ (or $\delta=0$ ) and then from (22) that

$$
p(t)\{\alpha f(t)-\gamma g(t)\}\{h(t)-\rho\}=0 \quad \text { a.e. on } E,
$$

if $\rho=\zeta$. When $\alpha \gamma \neq 0$, the same conclusion is valid if $\rho=\varepsilon+\zeta$, in which $\varepsilon=\alpha \delta / \gamma=\beta \gamma / \alpha$. We define $E_{7}$ as the subset of $E-E_{1}$ on which $h(t) \neq \rho$. If meas $E_{7}=0$, then (6) is surely true, and if meas $\left(E-E_{1}-E_{7}\right)=0$, then (4) is surely true. Henceforth, assume that meas $E_{7}>0$, meas $\left(E-E_{1}-E_{7}\right)>0$. We then infer from (23) that $\alpha f(t)=\gamma g(t)$ a.e. on $E_{7}$. If we multiply (5) as an equality, by $\alpha$ and then by $\gamma$, a little algebra shows that

$$
\begin{aligned}
& p(t) g(t)\{h(t)-\rho\} p(u)\{\alpha f(u)-\gamma g(u)\}=0, \\
& p(t) f(t)\{h(t)-\rho\} p(u)\{\alpha f(u)-\gamma g(u)\}=0
\end{aligned}
$$

for almost all $(t, u)$ in $E_{7} \times\left(E-E_{1}-E_{7}\right)$. Because $h(t) \neq \rho$ on $E_{7}, p(u) \neq 0$ on $E-E_{1}$, and $p(t) g(t)$ and $p(t) f(t)$ cannot both vanish at any point $t$ in $E-E_{1}$, we conclude that $\alpha f(u)=\gamma g(u)$ a.e. on $E-E_{1}-E_{7}$. Hence $\alpha f(u)=\gamma g(u)$ a.e. on $E-E_{1}$. This is sufficient to prove (4), and to complete the proof of Theorem 2.

Although the elementary concept of monotonicity for a single function is meaningless for a general set $E$, we can say that the pair of measurable functions $h(t)$ and $k(t)$ are "monotone in the same sense on $E$," or "similarly ordered," when

$$
\{h(t)-h(u)\}\{k(t)-k(u)\} \geq 0 \quad \text { a.e. on } E^{2} .
$$

This concept generalizes that defined in $[4$, p. 10] when $E$ is an interval on the real line, and has been used in [3, p. 168].

Corollary 4. Suppose that $p(t) g(t)>0$ a.e. on $E$ and that the functions $h(t)=$ $\alpha f(t)+\gamma g(t)$ and $k(t)=f(t) / g(t)$ are monotone in the same sense on $E$. Then the inequality (1) is true and is strict if (24) is strict on a measurable subset of $E^{2}$ whose measure is positive. Finally, equality a.e. in (24) occurs if and only if either (21) holds a.e. on $E$ or (6) holds a.e. on E.

The inequalities (5) and (24) are equivalent, because $p(t) g(t)>0$ a.e. on $E$. Moreover, $\alpha>0$, the equalities (4) and (21) are equivalent, and meas $E_{1}=0$. Now the corollary follows at once from Theorem 2.

\section{A SPECIAL CASE}

If we rescale both the independent and the dependent variables in the special case mentioned in $\S 1$, it may be described as the case in which $v$ is a positive number, $E=(0, v), f(t)=t^{2} J_{0}^{\prime \prime}(t), g(t)=J_{0}(t)$, and $p(t)$ is integrable and positive a.e. on $E$. Then

$$
\begin{gathered}
f(t)<0 \quad \text { if } 0<t<j_{1}^{\prime} \sim 1.8412, \\
g(t)>0 \quad \text { if } 0<t<j_{0} \sim 2.4048, \\
g^{\prime}(t)=-J_{1}(t)<0 \quad \text { if } 0<t<j_{1} \sim 3.8317,
\end{gathered}
$$


in which $j_{n}$ and $j_{n}^{\prime}$ are the smallest positive zeros [1, pp. 409, 411] of $J_{n}$ and $J_{n}^{\prime}$, respectively, and $J_{n}$ is the Bessel function of the first kind and order $n$. Moreover, $f^{\prime}(t)=-t\left\{J_{0}(t)-t J_{1}(t)\right\}$. Hence [1, p. 414] the smallest positive zero of $f^{\prime}(t)$ is $v_{m} \sim 1.2558$, and

$$
f^{\prime}(t)<0 \quad \text { if } 0<t<v_{m} .
$$

It follows from (25) that $\left(f^{2} / g^{2}\right)^{\prime}=2 f\left(f^{\prime} g-f g^{\prime}\right) / g^{3}>0$ if $0<t<v_{m}$, so that

$$
\max _{t \in E}\left[\{f(t) / g(t)\}^{2}\right]=\{f(v) / g(v)\}^{2}=\left\{v^{2} J_{0}^{\prime \prime}(v) / J_{0}(v)\right\}^{2}
$$

if $0<v \leq v_{m}$. Moreover, $f^{\prime} / g^{\prime}>0$ if $0<t<v_{m}$ and

$$
\left(f^{\prime} / g^{\prime}\right)^{\prime}=-2 t-t\left\{J_{1}^{2}(t)-J_{0}(t) J_{2}(t)\right\} / J_{1}^{2}(t)<0,
$$

if $0<t<j_{1}$, because [5, Equation (1), p. 152]

$$
J_{1}^{2}(t)-J_{0}(t) J_{2}(t)=\left(8 / t^{2}\right) \sum_{n=1}^{\infty} n J_{2 n}^{2}(t)>0 .
$$

Therefore, $\left\{\left(f^{\prime} / g^{\prime}\right)^{2}\right\}^{\prime}=2\left(f^{\prime} / g^{\prime}\right)\left(f^{\prime} / g^{\prime}\right)^{\prime}<0$ if $0<t<v_{m}$, and

$$
\min _{t \in E}\left[\left\{f^{\prime}(t) / g^{\prime}(t)\right\}^{2}\right]=\left\{f^{\prime}(v) / g^{\prime}(v)\right\}^{2}=v^{2}\left[v-\left\{J_{0}(v) / J_{1}(v)\right\}\right]^{2}
$$

if $0<v \leq v_{m}$. It follows from (26) and (28) that (23) is true when $0<v \leq v_{m}$ if and only if $\left\{J_{0}^{2}(v)-J_{1}^{2}(v)\right\} \Phi(v) \geq 0$, in which $\Phi(v)=\left\{J_{1}(v) / J_{0}(v)\right\}+$ $\left\{J_{0}(v) / J_{1}(v)\right\}-2 v$. We infer from the identity [5, Equation (14), p. 152],

$$
v^{2}\left\{J_{0}^{2}(v)+J_{1}^{2}(v)\right\}=4 \sum_{n=0}^{\infty}(2 n+1) J_{2 n+1}^{2}(v),
$$

that $J_{0}^{2}(v)>J_{1}^{2}(v)$ when $v^{2} \leq 2$, so that (23) is true when $0<v \leq v_{m}$ if and only if $\Phi(v) \geq 0$. We observe that $\lim _{v \rightarrow 0+} \Phi(v)=+\infty, \Phi\left(v_{m}\right)=$ $\left(1-v_{m}^{2}\right) / v_{m}<0$, and

$$
\left.\Phi^{\prime}(v)=-2-\left\{J_{0}^{2}(v)-J_{1}^{2}(v)\right\}^{3} /\left\{J_{0}(v)+J_{1}(v)\right\} J_{0}(v) J_{1}(v)\right\}^{2}<0 .
$$

Therefore, (23) is true in the strict sense when $0<v \leq v_{m}$ if and only if $0<v<v_{1}$, in which $v_{1}$ is the unique zero of $\Phi(v)$ such that $0<v_{1}<v_{m}$. We find that $v_{1} \sim 1.0944$, and conclude from Corollary 3 that (1) holds when $0<v \leq v_{1}$.

We have not been able to use Theorem 1 or any of its corollaries to improve this result, even in the very special case when $p(t)=t$. On the other hand, it follows from (25) that $(f / g)^{\prime}<0$ if $0<t<j_{1}$. Hence the hypotheses of Corollary 4 are satisfied if $h^{\prime}(t)=\alpha f^{\prime}(t)+\gamma g^{\prime}(t)<0$ when $0<t<v<j_{0}$, or because $\alpha>0$ and $g^{\prime}(t)<0$ when $0<t<v<j_{1}^{\prime}$, if $f^{\prime}(t) / g^{\prime}(t)>-\gamma / \alpha$ 
when $0<t<v<j_{1}^{\prime}$. It follows from (27) that this last condition holds if and only if

$$
\Psi(v)=\left\{f^{\prime}(v) / g^{\prime}(v)\right\}+\int_{0}^{v} p(t) f(t) d t / \int_{0}^{v} p(t) g(t) d t \geq 0 .
$$

It is easy to see that $\Psi\left(v_{1}\right)>0>\Psi\left(v_{m}\right)$ and that $\Psi^{\prime}(v)=\left\{f^{\prime}(v) / g^{\prime}(v)\right\}^{\prime}+$ $\alpha^{-2} G(v)$ a.e. when $0<v<v_{m}$, in which

$$
G(v)=p(v) g(v) \int_{0}^{v} p(t) g(t)[\{f(v) / g(v)\}-\{f(t) / g(t)\}] d t .
$$

Therefore, $\Psi^{\prime}(v)<0$ a.e. when $0<v \leq v_{m}$, and there is a unique $v_{2}$ such that $v_{1}<v_{2}<v_{m}$ for which $\Psi\left(v_{2}\right)=0$; moreover, $\alpha^{2} \delta-\gamma^{2} \beta>0$ when $0<v \leq v_{2}$.

When $p(t)=t$, the equation defining $v_{2}$ can be written in the form

$$
J_{0}\left(v_{2}\right)-2 v_{2} J_{1}\left(v_{2}\right)+3 J_{2}\left(v_{2}\right)=0 .
$$

We find that $v_{2} \sim 1.1668$.

\section{REFERENCES}

1. M. Abramowitz and I. A. Stegun, eds., Handbook of mathematical functions with formulas, graphs, and mathematical tables (tenth printing), Wiley \& Sons, New York, 1972.

2. E. F. Beckenbach and R. Bellman, Inequalities, Springer-Verlag, New York, 1961.

3. G. H. Hardy, J. E. Littlewood, and G. Polya, Inequalities, Cambridge Univ. Press, Cambridge, 1934.

4. D. S. Mitrinovic, Analytic inequalities, Springer-Verlag, New York, 1970.

5. G. N. Watson, A treatise on the theory of Bessel functions, 2nd ed., Cambridge Univ. Press, Cambridge, 1944.

6. J. E. Wilkins, Jr., Apodization for maximum Strehl criterion and specified Sparrow limit of resolution for coherent illumination, J. Optical Soc. Amer. 67 (1977), 553-557.

Department of Mathematics, Clark Atlanta University, Atlanta, Georgia 30314 